OPEN

SUBJECT AREAS:

ELECTRONIC PROPERTIES

AND MATERIALS

STRUCTURAL PROPERTIES

Received

1 September 2014

Accepted

18 November 2014

Published

15 December 2014

Correspondence and requests for materials should be addressed to

L.L. (luying.li@hust. edu.cn) or J.W.

(wang@whu.edu.cn)

\section{Polarity continuation and frustration in ZnSe nanospirals}

\author{
Luying Li', Fanfan Tu', Lei Jin², Wallace C. H. Choy ${ }^{3}$, Yihua Gao ${ }^{1,4}$ \& Jianbo Wang ${ }^{2}$
}

\begin{abstract}
${ }^{1}$ Center for Nanoscale Characterization and Devices, Wuhan National Laboratory for Optoelectronics, Huazhong University of Science and Technology, Wuhan 430074, China, ${ }^{2}$ School of Physics and Technology, Center for Electron Microscopy and MOE Key Laboratory of Artificial Micro- and Nano-structures, Wuhan University, Wuhan 430072, China, ${ }^{3}$ Department of Electrical and Electronic Engineering, University of Hong Kong, Pokfulam Road, Hong Kong, China, ${ }^{4}$ School of Physics, Huazhong University of Science and Technology, Wuhan 430074, China.
\end{abstract}

ZnSe nanospirals including structures with polarity continuation and polarity frustration are simultaneously observed at atomic resolution. Through careful analysis of polarity within each dumbbell based on aberration-corrected high-angle annular-dark-field imaging, polarity continuation across parallel polytype interfaces as well as the surrounding Z-shape faulted dipoles is verified. Moreover, polarity frustration across regions with different stacking sequence, which would lead to accumulations of boundary interface charges in the triangular-shaped mixed regions with potential optoelectronic applications, is carefully studied.

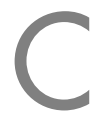

ompound semiconductors composed of III-V or II-VI elements have been highlighted as excellent candidates for a wide range of electric and optoelectronic devices. The partial ionicity of the chemical bonds between the different elements of the compound, normally referred to as "dumbbells", leads to the formation of atomic pairs of opposite partial charge. The overall polarity is related to the dipole moment arised from those partial charges of dumbbells. Apart from acting as the driving force for unidirectional growth ${ }^{1-2}$, the polarity would introduce macroscopic polarization effects in structures without centrosymmetry of the tetrahedral bonds. For compound semiconductors, the typical polytype structures include the zincblende (ZB) with cubic stacking in the [111] direction, and wurtzite (WZ) with hexagonal stacking in the [0001] direction. With controlled growth of compound semiconductor nanowires (NWs) with alternating ZB and WZ segments ${ }^{3-4}$, charge redistributions in certain polytype regions would happen due either to band offsets at the polytype interfaces ${ }^{5-6}$, or spontaneous polarization in polytype regions with asymmetric structures ${ }^{7-10}$.

The polarity maintains the same direction across the parallel polytype interfaces within the semiconductor NWs, as has been shown in many cases ${ }^{7-8,11}$. On the other hand, the reversal of polarity across interfaces is also possible in various semiconductors. The periodically polarity-inverted $\mathrm{ZnO}$ heterostructures on (0001) $\mathrm{Al}_{2} \mathrm{O}_{3}$ reveal strong exitonic emission at the interface region ${ }^{12}$. Polarity inversion of $\mathrm{GaN}$ is realized by deposition and patterning of a thin low-temperature AlN nucleation layer ${ }^{13}$. An epilayer-thickness-dependent polarity inversion for the growth of $\mathrm{CdTe}$ on $\mathrm{Sb}(\mathrm{Bi}) / \mathrm{CdTe}(111) B$ is observed with the significance of "polarity engineering" 14 . The InAs/single-layer graphene/InAs double heterostructure also exhibits polarity inversion ${ }^{15}$, which offers the opportunities for doping effect ${ }^{16}$ investigations with functional device applications.

In this current study, polarity continuation as well as polarity frustration is simultaneously observed within ZnSe nanospirals. The chemical information is determined using scanning transmission electron microscopy (STEM), with electron- energy-loss spectroscopy (EELS) elemental mapping. The local polarity is revealed by aberration-corrected high-angle annular-dark-field (HAADF) imaging, and possible atomic models are constructed for two different cases of polarity frustration.

\section{Results and Discussion}

The ZnSe nanospirals are bent into spiral-like shapes with relatively rougher inner side surfaces, as represented by the SEM image in Figure 1a and HAADF image in Figure 1b. The nanospirals are easily broken into small pieces with belt-like shape during TEM sample preparation due to their large sizes. The growth direction is along $<-211>$ axis, and the [111] axes are always pointing towards the center of the nanospiral. There is a high density of stacking faults on $\{111\}$ planes featured by the parallel dark lines along the growth direction in Figure 1c. Two typical regions are framed by red and blue circles, and the high resolution transmission electron microscopy 

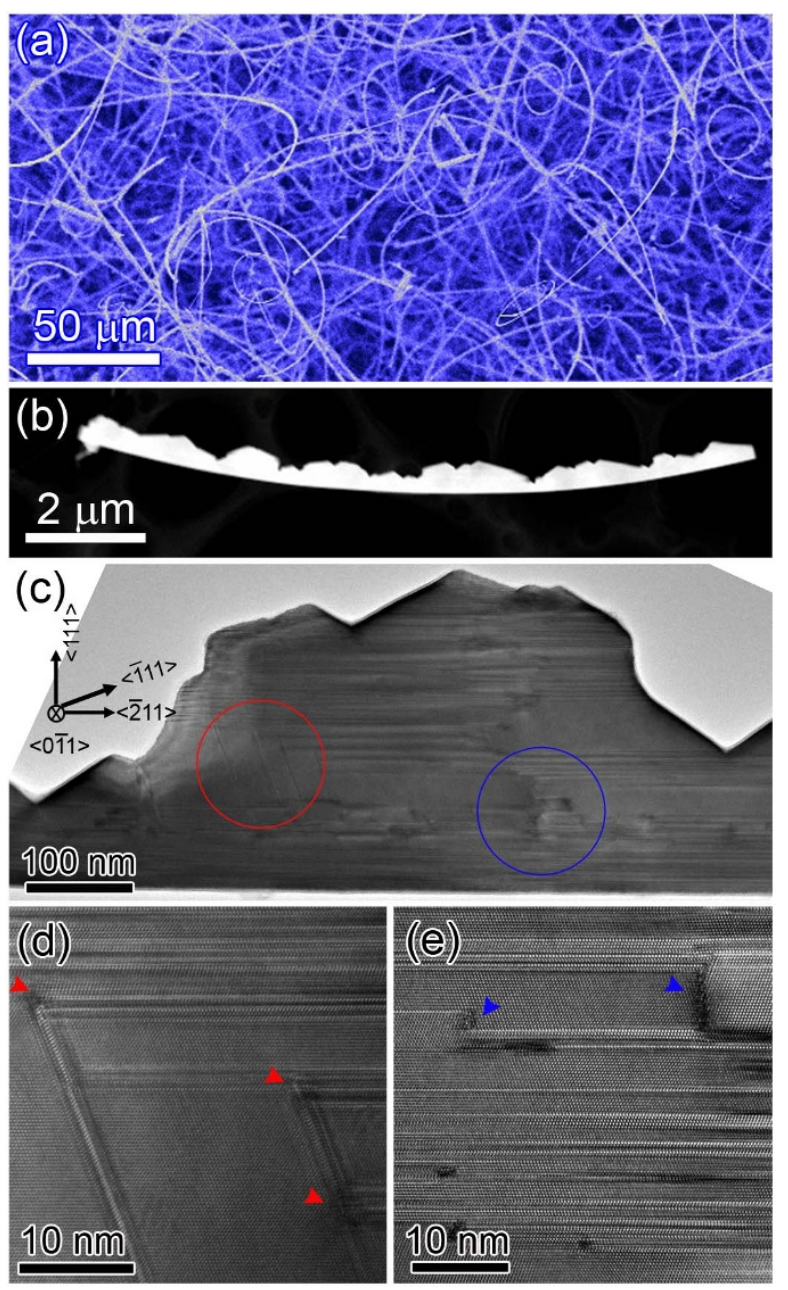

Figure $1 \mid$ Overall features of ZnSe nanospirals. (a) SEM image of ZnSe nanospirals. (b) HAADF image of a broken piece of ZnSe nanospirals with much rougher inner side surfaces. (c) TEM image of part of a selected nanospiral with parallel dark lines along the growth direction. Two featured regions are framed by red and blue circles, and the HRTEM images with similar features are presented in (d) and (e), respectively. The locations of Lomer-Cottrell partial dislocations in (d) are indicated by red arrows, and the mixed regions of different stacking sequence in (e) are labeled by blue arrows.

(HRTEM) images with similar features are presented in Figures 1de, respectively. Figure 1d shows several stacking faults on the $\{111\}$ planes intersecting stacking faults on the $\{-111\}$ planes, forming the so-called Z-shape faulted dipoles. Lomer-Cottrell partial dislocations would form at the intersection points, as indicated by the red arrows, which are reported to be responsible for the spiral-like morphology of the $\mathrm{ZnSe}$ nanospirals ${ }^{17}$. Figure 1e also shows many stacking faults on the $\{111\}$ planes. The blue arrows indicate mixed regions where the two sides have different stacking sequence. The detailed structural information at those regions are not obtainable due to the limited resolution of the microscope used for HRTEM imaging, and the unavoidable image delocalization effect that occurs in a microscope without aberration correction for the imaging mode.

STEM EELS is a powerful chemical analysis technique which enabled the chemical identification of individual atoms in hexagonal $\mathrm{BN}$ monolayers containing substitutional defects ${ }^{18}$. Figures $2 \mathrm{a}-\mathrm{b}$ are STEM-EELS elemental maps for $\mathrm{Zn}$ and Se, respectively. The bright contrast indicates the exact atomic column locations of the selected elements. Thus, chemical information is determined at atomic resolution. Figure $2 \mathrm{c}$ is the combined elemental map obtained by over-
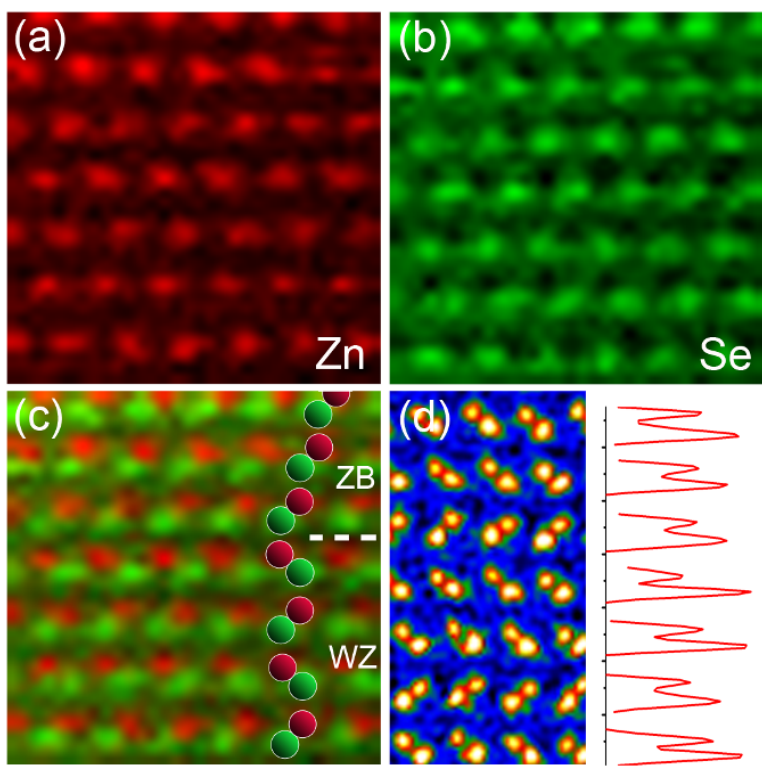

Figure $2 \mid$ Chemical information of the nanospirals at atomic resolution, and the verification of polarity continuation across parallel polytype interfaces. (a) and (b) are STEM EELS elemental maps of Zn and Se for specific region of $\mathrm{ZnSe}$ nanospiral. The bright contrast indicates the locations of the selected atomic columns. (c) The overall elemental map obtained by overlapping (a) and (b). The red and green balls represent $\mathrm{Zn}$ and Se column positions, respectively. The interface of $\mathrm{ZB}$ and $\mathrm{WZ}$ structures are labeled by a white dashed line. (d) (Left) Aberrationcorrected HAADF image of another region including variable stacking sequence. (Right) Intensity line profile of one line of dumbbells from top to bottom.

lapping Figures $2 \mathrm{a}$ and $2 \mathrm{~b}$. Red and green balls are added to indicate the $\mathrm{Zn}$ and Se atomic column positions. The dumbbells change stacking sequence from $\mathrm{ZB}$ to $\mathrm{WZ}$ from top to bottom, where the $\mathrm{ZB} / \mathrm{WZ}$ interface is labeled by a white dashed line. It is clear that the $\mathrm{Zn}$ columns (red balls) are always on the top side of the dumbbells across the interface.

Figure $2 \mathrm{~d}$ (left) is an aberration-corrected HAADF image of another region of the same nanospiral. The dumbbells are clearly resolved at atomic resolution, and change stacking sequence several times from top to bottom. It is well known that the HAADF intensity is proportional to projected sample thickness via the factor $Z^{\alpha}$, where $\mathrm{Z}$ is the atomic number, and $\alpha$ should have a value between 1 and 2 depending on the microscope geometry ${ }^{19}$. Thus, heavier atoms are expected to have brighter contrast. Figure $2 \mathrm{~d}$ (right) is the intensity line profile of one line of dumbbells indicating that, even with complicated stacking sequence variations, the top atoms always have lower intensities. Thus, both the STEM EELS elemental mapping and aberration-corrected HAADF imaging confirm that the polarity continuation is maintained across parallel polytype interfaces in $\mathrm{ZnSe}$, similar to cases previously reported for other compound semiconductor $\mathrm{NWs}^{11}$.

The Z-shape faulted dipole was first reported in 1963 as faint straight lines in strong-beam electron micrographs of deformed f.c.c. metals ${ }^{20}$. The atomic structure of the central stacking faults of a Z-shape faulted dipole in deformed GaAs was determined based on HRTEM imaging and image simulations ${ }^{21}$. The atomic configurations at InAs partial dislocation cores associated with Z-shape faulted dipoles were observed directly using aberration-corrected HAADF imaging $^{22}$. Figure $3 \mathrm{a}$ is aberration-corrected HAADF image of a typical Z-shape faulted dipole within ZnSe nanospiral. The shear distortions present in the image have been removed according to the perfect ZB structure in [0-11] projection, and the high frequency 

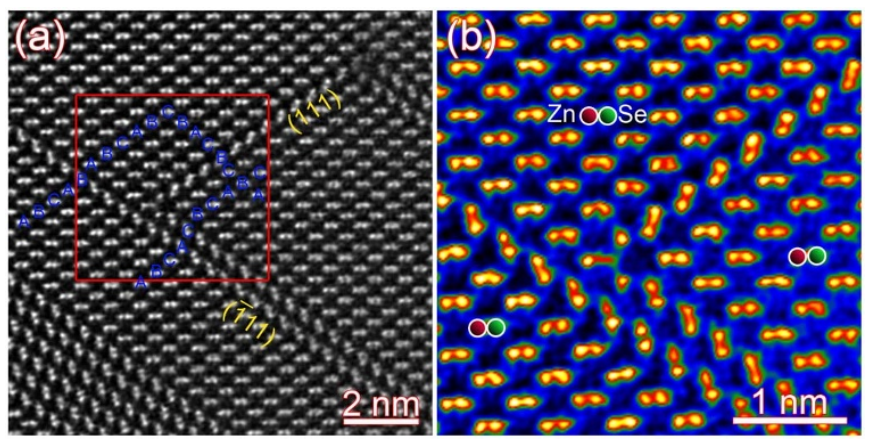

Figure 3 Polarity continuation surrounding dislocation cores formed by intersecting stacking faults. (a) Aberration-corrected HAADF image of a Z-shape faulted dipole within ZnSe nanospiral, and the corresponding stacking sequence is overlapped with blue symbols. One of the dislocation core region framed in a red box is magnified and shown in pseudo-color in (b). Single atomic columns at the dislocation core and the dumbbells in the surrounding regions are clearly resolved. Polarity continuation is observed across both of the (111) and (-111) stacking faults planes.

noise has been filtered using the annular mask tool in Digital Micrograph.

Stacking sequence analysis in Figure 3 a reveals that the dislocation core framed by the red box is formed at the intersection of one intrinsic stacking fault in (111) plane and the other intrinsic stacking fault in $(-111)$ plane. The leading $30^{\circ}$ partial dislocation $(C \delta)$ dissociated from a perfect dislocation lying in a (111) plane, reacts with a stacking fault lying in a $(-111)$ plane, and generates a stair-rod $(\alpha \delta)$ and another partial dislocation $(\mathrm{C} \alpha)$. When gliding in the $(-111)$ stacking fault plane, the latter partial dislocation would transform the intrinsic stacking fault into an extrinsic stacking fault. The formation mechanism could be expressed applying Thompson's notation as: $\mathrm{C} \delta$ $\rightarrow \mathrm{C} \alpha+\alpha \delta$, which resembles the case of dislocation core formation in InAs nanopillars ${ }^{22}$.

Figure $3 b$ is magnified image of the region in the red box in Figure 3 a shown in pseudo-color to enhance the contrast. The dumbbell structures are clearly resolved with the heavier atomic columns always on the right side, as indicated by the red and green balls in all three regions divided by the stacking faults in $(111)$ and $(-111)$ planes. Thus, the existence of stacking faults does not break polarity continuation across stacking fault planes no matter in which planes the stacking faults are lying. It should be noted that at the locations within or close to the dislocation core, the polarity determination may lead to ambiguous results, which might be due to the fact that $\mathrm{Zn}$ and Se have very close atomic numbers (Zn:30, Se:34), and the corresponding $\mathrm{Zn}$ and Se columns have relatively close image intensities. In that case, local strain might have effects great enough to reverse the relative intensities so that the actual atom types are not what they appear to be.

All of the above are examples of polarity continuation. Moreover, cases of polarity frustration are also observed within the same $\mathrm{ZnSe}$ nanospiral, such as the regions indicated by blue arrows in Figure $1 \mathrm{~d}$. The aberration-corrected HAADF image shown in Figure 4a is one such case. As before, this region includes plenty of parallel polytype interfaces, i.e., parallel stacking faults. However, the left and right hand sides have different stacking sequence. The dumbbells just above the white dashed line have the same polarity (along $<-111>$ direction) from left to right. The dumbbells on the right hand side retain the same polarity down to bottom, while those just above the red dashed line on the left hand side change stacking sequence, i.e., the vertical polarity component keeps the same direction, and the horizontal polarity component is reversed from left to right, which resembles the situation surrounding the (112) symmetric lateral twin with mirror symmetric crystal polarity ${ }^{23}$. Thus, this

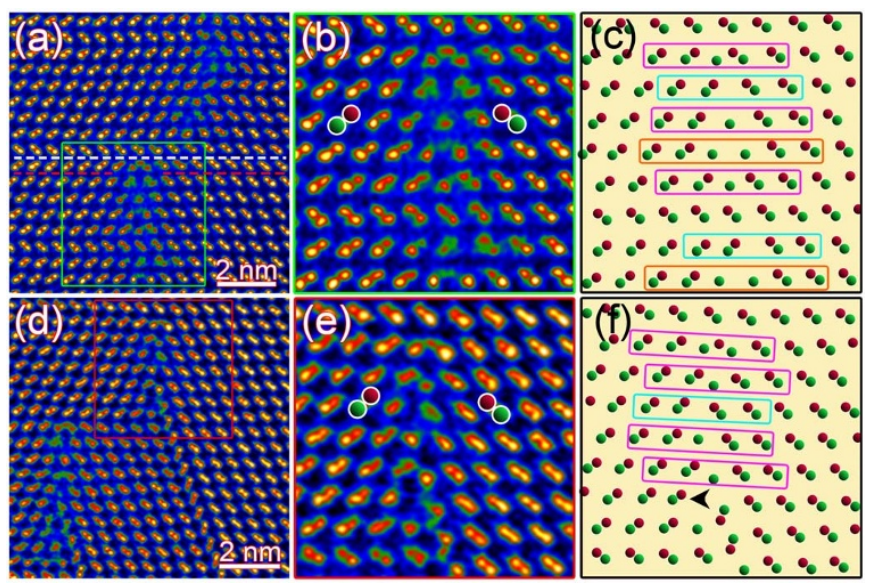

Figure $4 \mid$ Polarity frustration across the triangular regions with different stacking sequence on both sides. (a) Aberration-corrected HAADF image of specific region in $\mathrm{ZnSe}$ nanospiral including polarity frustration. The dumbbells just above the white dashed line are of the same polarity, while those just above the red dashed line show reversed polarity in the

horizontal direction. (b) The magnified image of the region framed by the green box in (a). (c) The structural model constructed based on (b). (d) Aberration-corrected HAADF image of another region including polarity frustration as well as different number of rows on both sides of the mixed region. (e) The magnified image of the region framed by the red box in (d). (f) The structural model constructed based on (e), and the black arrow labels the location of an extra half plane on the left hand side. The red and green balls in (b) and (e) represent $\mathrm{Zn}$ and Se atomic columns, respectively. The rectangular boxes in (c) and (f) indicate different configurations of polarity frustration.

case is described as polarity frustration, rather than polarity reversal. As this trend continues, a triangular region is formed where the left and right parts meet.

The magnified image of the triangular regions framed by the green box in Figure 4a is shown in Figure $4 \mathrm{~b}$. The polarity is easily determined from the relative intensities within each dumbbell. It is clear that the $\mathrm{Zn}$ columns are always on the top along the vertical direction, which is the case for all of the parallel polytype interfaces. However, in the horizontal direction, the $\mathrm{Zn}$ columns are pointing to the right for the left part, and have reversed direction for the right part. Unpaired atomic columns appear in the mixed region where the atom types can be determined by extrapolating from the surrounding perfect crystal $^{24}$. A structural model is constructed based on the aberration-corrected HAADF image and presented in Figure 4c, where the red and green balls correspond to $\mathrm{Zn}$ and Se columns, respectively. Although the stacking sequence is quite different for both sides of the mixed region, the same number of rows is maintained from left to right. Thus, distortions of lattices surrounding the mixed region are not obvious.

There are also regions with both different stacking sequence and numbers of rows from left to right across the mixed region, as can be seen from the region framed by the red box in Figure $4 \mathrm{~d}$. Figure $4 \mathrm{e}$ is the magnified image of the boxed region in Figure $4 \mathrm{~d}$. While the right hand side retains the same stacking sequence from top to bottom, the stacking sequence on the left hand side changes from $\mathrm{ZB}$ to $\mathrm{WZ}$ for one period, which results in reversed polarity components in the horizontal direction. The same transition reoccurs at the bottom of the left hand side region, and the horizontal component of polarity changes back to the original, which is the same as the right hand side region. The mixed region also shows unpaired atomic columns with variable bonding lengths to the neighbouring atoms columns.

Figure $4 \mathrm{f}$ is the structural model constructed according to Figure 4e. As compared to the model in Figure 4c, an extra half plane 
is inserted in the left hand side region in Figure $4 \mathrm{f}$ indicated by a black arrow, which leads to an overall upward bending of the lattices above the extra half plane. On the other hand, the right hand side region is not quite disturbed by the lattice distortions on the left. In addition, an intrinsic stacking fault starts to form below the extra half plane and extends down to the bottom of the field of view in Figure $4 \mathrm{~d}$.

A dipole can be considered as a charge distribution with negative charges on one side and positive charges on the other side. If all the dipoles in a material are aligned, the charges inside will be canceled out, i.e. the negative charges on one dipole will be canceled by the positive charges on its neighbouring dipole except at the surfaces where no cancellation is possible. The effect of polarization is to create a bound charge over the surface of the material (bound surface charge $)^{25}$. In the current study, the dumbbell is composed of $\mathrm{Zn}^{2+}$ and $\mathrm{Se}^{2-}$ ions, which have polarity components in both vertical and horizontal directions. While the vertical polarity components always maintain the same direction, the horizontal components reverse their directions in some regions, as can be seen from Figures $4 \mathrm{~b}$ and $4 \mathrm{e}$.

Careful analysis of the structures at the mixed regions reveals that there are three common configurations for polarity frustration, as presented in the purple, cyan, and orange boxes in Figures $5 \mathrm{a}-\mathrm{c}$. The red and green circles represent equivalent positive and negative charges, the black arrows indicate the formation of dipoles in the horizontal direction, and the blue boxes include pairs of opposite charges which would be canceled out leaving no net charge. The configuration in Figure 5a has one unpaired $\mathrm{Se}^{2-}$ in the mixed region, and the cancellation of opposite charges head to end would still leave positive charges from the $\mathrm{Zn}^{2+}$ indicated by the red ball, which could be considered as "bound interface charge", as opposed to "bound surface charge" mentioned above. In the same way, the configuration in Figure $5 \mathrm{~b}$ would have net positive charges from two $\mathrm{Zn}^{2+}$ columns with no cancellation possible, and the configuration in Figure $5 \mathrm{c}$ has no net charge left in the mixed region.

The three configurations of polarity frustration are labeled by colored boxes in Figure $4 \mathrm{c}$ and $4 \mathrm{f}$, where the rows with purple and cyan boxes would have excess positive charges accumulated in the mixed region, and the rows with orange boxes or no polarity frustration would have no excess charge of either type. Thus, the formation of a triangular region with polarity frustration on both sides would lead to excess charge accumulation in the mixed region. With controlled growth of structures with periodical variations of

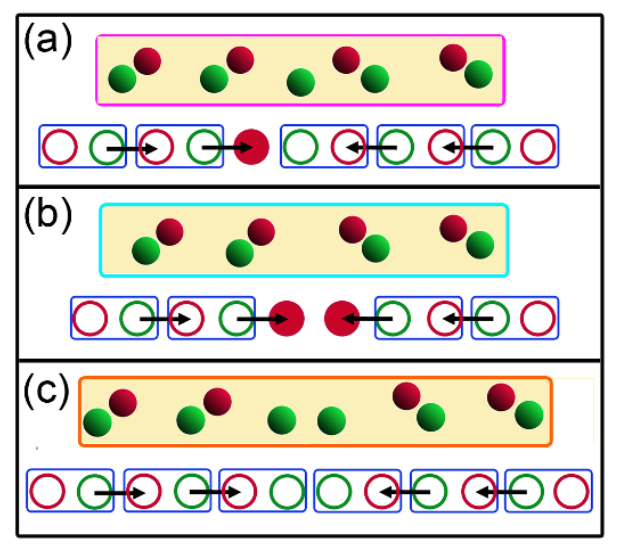

Figure 5 | Three possible configurations of polarity frustration within the mixed regions. The purple, cyan, and orange boxes include structural models of the three configurations labeled as (a), (b) and (c), respectively. The red and green balls represent $\mathrm{Zn}$ and Se columns. The red and green circles represent equivalent positive and negative charges, the black arrows indicate the formation of dipoles in the horizontal direction, and the blue boxes include pairs of opposite charges which would be canceled out leaving no net charge. polarity frustration, tailoring of charge distributions in semiconductor nanostructures becomes possible, in company with other ways of controlling charge distributions via band structure engineering at heterostructural interfaces ${ }^{26-27}$ and polarization fields at polytype interfaces ${ }^{7}$. Moreover, it is reported that single $\mathrm{Cd}$ and Te columns at intragrain Schockley partial dislocation cores where stacking faults terminate, cause significant energy band-bending, which is responsible for the improved cell efficiency of CdTe based solar cells ${ }^{28}$. The existence of unpaired Se columns in the mixed region might also have impact on the corresponding band structures with possible optoelectronic applications: this requires further density-functional theory calculations.

\section{Conclusions}

In summary, structures with polarity continuation and polarity frustration are simultaneously observed within $\mathrm{ZnSe}$ nanospirals. Both parallel polytype interfaces and Z-shape faulted dipoles formed by the intersection of stacking faults in different $\{111\}$ planes maintain polarity across the interfaces. On the other hand, polarity frustration is formed in the triangular-shaped mixed region with different stacking sequence on both sides. While the vertical component of polarity is maintained throughout the whole region, the horizontal component reverses direction across the mixed region, which would result in excess charge accumulation in that region. Our results suggest that through careful control of crystal structures of compound semiconductors, tailoring of charge distributions is possible via polarity frustration, which enables potential optoelectronic applications.

\section{Methods}

The ZnSe nanospirals were grown through heating $\mathrm{ZnSe}$ powder in a tube furnace. The growth process was divided into two stages during which the reaction pressure was varied. Detailed growth information has been published elsewhere ${ }^{17}$. Slight variations of the growth parameters result in quite different morphologies ${ }^{29-31}$. The yellowish products were scratched off the substrate, sonicated in ethanol, and then dropped onto the holey carbon-coated copper grid for TEM observation. The HRTEM images and aberration-corrected HAADF images were obtained using a Titan G2 60-300 kV electron microscope with probe correction operated at an acceleration voltage of $300 \mathrm{kV}$. The STEM EELS elemental mapping were carried out using a JEOL ARM200F electron microscope with probe correction operated at $200 \mathrm{kV}$.

1. Uccelli, E. et al. Three-dimensional multiple-order twinning of self-catalyzed GaAs nanowires on Si substrate. Nano Lett. 11, 3827-3832 (2011).

2. Persson, A. I. et al. Solid-phase diffusion mechanism for GaAs nanowire growth. Nat. Mater. 10, 677-681 (2004)

3. Caroff, P. et al. Controlled polytypic and twin-plane superlattices in III-V nanowires. Nat. Nanotechnol. 4, 50-55 (2009).

4. Dick, K. A., Thelander, C., Samuelson, L. \& Caroff, P. Crystal phase engineering in single InAs nanowires. Nano Lett. 10, 3494-3499 (2010).

5. Akopian, N., Patriarche, G., Liu, L., Harmand, J. \& Zwiller, V. Crystal phase quantum dots. Nano Lett. 10, 1198-1201 (2010).

6. Spirkoska, D. et al. Structural and optical properties of high quality zinc-blende/ wurtzite GaAs nanowire heterostructures. Phys. Rev. B 80, 245325 (2009).

7. Li, L. et al. Polarization-induced charge distribution at homogenous zincblende/ wurtzite heterostructural junctions in ZnSe nanobelts. Adv. Mater. 24, 1328-1332 (2012).

8. Li, L. et al. Determination of polarization-fields across polytype interfaces in InAs nanopillars. Adv. Mater. 26, 1052-1057 (2014).

9. Georgescu, L., Gevaux, D., Klopper, A., Verberck, B. \& Wright, A. Research highlights-Material mix. Nature Phys. 9, 753 (2013).

10. Dayeh, S. A., Susac, D., Kavanagh, K. L., Yu, E. T. \& Wang D. Structural and roomtemperature transport properties of zinc blende and wurtzite InAs nanowires. Adv. Funct. Mater. 19, 2102-2108 (2009).

11. Mata, M. et al. Polarity assignment in $\mathrm{ZnTe}, \mathrm{GaAs}, \mathrm{ZnO}$, and $\mathrm{GaN}-\mathrm{AlN}$ nanowires from direct dumbbell analysis. Nano Lett. 12, 2579-2586 (2012).

12. Park, J. S. et al. Structural and optical investigations of periodically polarity inverted $\mathrm{ZnO}$ heterostructures on (0001) $\mathrm{Al}_{2} \mathrm{O}_{3}$. Appl. Phys. Lett. 94, 141904 (2009).

13. Liu, F. et al. The mechanism for polarity inversion of GaN via a thin AlN layer: direct experimental evidence. Appl. Phys. Lett. 91, 203115 (2007).

14. Cho, S. et al. Polarity inversion in polar-nonpolar-polar heterostructures. Phys. Rev. Lett. 87, 126403 (2001).

15. Hong, Y. et al. Van der Waals epitaxial double heterostructure: InAs/single-layer graphene/InAs. Adv. Mater. 25, 6847-6853 (2013). 
16. Kim, Y., Heben, M. J. \& Zhang, S. B. Nanotube wires on commensurate InAs surfaces: binding energies, band alignments, and bipolar doping by the surfaces. Phys. Rev. Lett. 92, 176102 (2004).

17. Jin, L., Wang, J. \& Choy, W. C. H. Growth of ZnSe nanospirals with bending mediated by Lomer-Cottrell sessile dislocations through varying pressure. Cryst. Growth Des. 8, 3829-3833 (2008).

18. Krivanek, O. L. et al. Atom-by-atom structural and chemical analysis by annular dark-field electron microscopy. Nature 464, 571-574 (2010).

19. Hartel, P., Rose, H. \& Dinges, C. Conditions and reasons for incoherent imaging in STEM. Ultramicroscopy 63, 93-114 (1996).

20. Mader, S. Electron Microscopy and Strength of Crystals (Inter-science, New York, 1963).

21. Lim, S., Shindo, D., Yonenaga, I., Brown, P. D. \& Humphreys, C. J. Atomic arrangement of a Z-shape faulted dipole within deformed GaAs. Phys. Rev. Lett. 81, 5350-5353 (1998).

22. Li, L. et al. Atomic configurations at InAs partial dislocation cores associated with Z-shape faulted dipoles. Sci. Rep. 3, 3229 (2013).

23. Cohen, D., McKernan, S. \& Carter, C. B. Characterization of the absolute crystal polarity across twin boundaries in gallium phosphide using convergent-beam electron diffraction. Microsc. Microanal. 5, 173-186 (1999).

24. Li, C. et al. Grain-boundary-enhanced carrier collection in CdTe solar cells. Phys. Rev. Lett. 112, 156103 (2014).

25. Griffiths, D. J. Introduction to electrodynamics (Prentice Hall, New Jersey, 1999, Chapter 4).

26. Li, L. et al. Observation of hole accumulation in Ge/Si core/shell nanowires using off-axis electron holography. Nano Lett. 11, 493-497 (2011).

27. Li, L., Ketharanathan, S., Drucker, J. \& McCartney, M. R. Study of hole accumulation in individual germanium quantum dots in p-type silicon by off-axis electron holography. Appl. Phys. Lett. 94, 232108 (2009).

28. Li, C. et al. Carrier separation at dislocation pairs in CdTe. Phys. Rev. Lett. 111, 096403 (2013)

29. Jin, L. et al. $\{113\}$ twinned ZnSe bicrystal nanobelts filled with $<111>$ twinnings. J. Phys. Chem. C 112, 4903-4907 (2008).

30. Jin, L. et al. Twinning mediated growth of ZnSe tri- and bi-crystal nanobelts with single crystalline wurtzite nanobelts as building blocks. CrystEngComm, 12, $150-158$ (2010).
31. Jin, L. et al. ZnSe heterocrystalline junctions based on zinc blende-wurtzite polytypism. J. Phys. Chem. C 114, 1411-1415 (2010).

\section{Acknowledgments}

This study was supported by National Natural Science Foundation of China (51371085, 11304106), MOE Doctoral Fund (20120142120059), the Fundamental Research Funds for the Central Universities (HUST: 2012QN107), SRF for ROCS, SEM, and the Natural Science Foundation of Hubei Province (2013CFB183). J. Wang acknowledges support from 973 Program (2011CB933300), National Natural Science Foundation of China (51271134, J1210061), the Fundamental Research Funds for the Central Universities, and the CERS-1-26 (CERS-China Equipment and Education Resources System). The authors thank Dr. E. Okunishi of JEOL for EELS experimental support, and Prof. David Smith for fruitful discussions and help in revising the manuscript.

\section{Author contributions}

L.L. carried out the TEM experiments and wrote the paper, L.J. and W.C. assisted the material growth, L.L. and J.W. designed the experiment. L.L., F.T., Y.G. and J.W. contributed to the data analyses and paper revisions.

\section{Additional information}

Competing financial interests: The authors declare no competing financial interests.

How to cite this article: Li, L. et al. Polarity continuation and frustration in $\mathrm{ZnSe}$ nanospirals. Sci. Rep. 4, 7447; DOI:10.1038/srep07447 (2014).

This work is licensed under a Creative Commons Attribution-NonCommercialNoDerivs 4.0 International License. The images or other third party material in this article are included in the article's Creative Commons license, unless indicated otherwise in the credit line; if the material is not included under the Creative Commons license, users will need to obtain permission from the license holder in order to reproduce the material. To view a copy of this license, visit http:// creativecommons.org/licenses/by-nc-nd/4.0/ 